\title{
11. Interview mit Dr. Michael Henker, ICOM Deutschland-Präsident 2014-2016
}

\subsection{Interviewtext}

\author{
Dr. Michael Henker im Interview zu den Herausforderungen für ICOM \\ Deutschland während seiner Amtszeit als Präsident (20I4-20I6), durchge- \\ führt von Tanja Leiminger am I9. Mai 2020 in München:
}

Die Jahre meiner Präsidentschaft von ICOM Deutschland - von 2014 bis 2016 - waren sowohl innenpolitisch als auch auf internationaler Ebene sehr interessant und brisant. Beide Aspekte beeinflussten die Verbandsarbeit und speziell meine Rolle als Präsident stark.

Zum einen manifestierte sich in der deutschen Innenpolitik zunehmend die Auffassung, dass Kultur und Museen konstitutiv für den Zusammenhalt der Gesellschaft sind. Viele Politiker hatten dieses Bewusstsein längst entwickelt, andere schoben das jedoch gerne beiseite. Durch die jüngst durchgeführte Bundestags- und Landtagswahl in Bayern traten viele neue politische Akteure ihre Ämter an, die es immer wieder von Neuem von der Wichtigkeit kultureller Arbeit zu überzeugen galt. Die neu gewählten Ansprechpartner waren im Bund Frau Prof. Monika Grütters und in Bayern Herr Dr. Ludwig Spaenle.

Zum anderen blieb auch in Deutschland die Kultur von internationalen Ereignissen nicht unbelastet. Wir befanden uns inmitten der Planungen der 20I3 beschlossenen Jahrestagung zum Thema >Museums Politics and Power<, von ICOM Deutschland zusammen mit ICOM USA und ICOM Russland, als die instabile politische Situation in der Ukraine zur russischen Annexion der Krim führte. Aus dem ukrainischen Nationalkomitee sowie auf internationaler Ebene erhoben sich kurzfristig Stimmen gegen eine Tagung mit ICOM Russland als Partner. Im Vorstand kamen wir jedoch letztendlich zu dem Beschluss, an der Durchführung der Tagung festzuhalten. Der Dialog auf der Ebene des Kulturaustausches sollte unabhängig von der politischen Situation der beteiligten Länder unbedingt weitergeführt werden. Durch unsere Argumentation konnten wir selbst erreichen, dass ICOM USA - die gegenüber Russland viel größerem politischen Druck ausgesetzt waren - weiterhin als Partner teilnimmt. Wir setzten den weltweiten Call for Papers fort und erhielten 340 Essays von denen wir etwa I20 sorgfältig ausgewählte Beiträge in vier 
Sektionen auf zwei Generalsitzungen aufteilten. Der erste Teil der Tagung fand vom 9. bis I2. September 20I4 in St. Petersburg in der Eremitage und in der Admiralität statt. Den zweiten Teil führten wir direkt im Anschluss an zwei Tagen (I3. und I4. September 20I5) in Jekaterinburg mit dem Schwerpunkt Industrielles Erbe und Technikmuseen durch. Rückblickend war die gemeinsame Tagung in Russland sehr erfolgreich und erhielt nicht zuletzt wegen der außerordentlichen Gesamtsituation weitreichende mediale Resonanz.

Mit etwas zeitlicher Verzögerung erreichten uns Fotografien, die das beschossene Museum des Donbass in Donezk zeigten und die nicht nur in Deutschland für Empörung sorgten. Bei den Pariser June Meetings 2015 bildeten wir anlässlich der akuten Bedrohung der Museen in der Ukraine durch diese prekäre politische Situation die ad hoc Gruppe >Museums in Danger<, die ich zusammen mit Martina Lehmannova, Präsidentin von ICOM CZ, leitete. Das vorübergehend einberufene Komitee setzte sich aus den ICOMNationalkomitees von Deutschland, Österreich, Tschechien, Polen, Weißrussland, der Slowakei, den baltischen Komitees und auch den Nationalkomitees der Konfliktparteien ICOM Ukraine und Russland zusammen. Im Rahmen eines großen Museumskongresses in Lodz, den wir mit den teilnehmenden ICOM-Komitees abhielten, versuchten wir, bei beiden Parteien um Verständnis für die dort entstandene Situation zu werben, denn dieser militärische und politische Konflikt löste auch innerhalb der Kulturwelt eine katastrophale Grundstimmung aus. Wir bemühten uns außerdem, mit Hilfe des damaligen Präsidenten des ICOM-Weltverbandes, Prof. Hans-Martin Hinz, die diplomatischen Möglichkeiten von ICOM International zu nutzen. Unsere Bestrebungen zur Verbesserung der Situation blieben jedoch ohne Resonanz. Weiterhin schwierig gestaltete sich das Vorhaben, den betroffenen Museen im Donbass Hilfsgüter und Hilfsleistungen zur Verfügung zu stellen, die aufgrund des Krieges geschlossen blieben. Von uns angebotene Ressourcen, wie etwa Drucker etc. wurden zu diesem Zeitpunkt schlichtweg nicht benötigt, da sie nicht einmal eingesetzt werden konnten.

Zeitgleich dazu ereignete sich 2015 das verheerende Erdbeben im Kathmandu Tal in Nepal. Dort konnte die Taskforce des ICOM-Weltverbandes vor Ort Hilfsgüter zur Verfügung stellen und Geldleistungen kanalisieren, woran wir uns - darunter auch ich persönlich - mit höchstem Engagement beteiligten.

Neben diesen einschneidenden politischen Ereignissen bildete ein weiterer Aspekt einen der inhaltlichen Schwerpunkte meiner Amtszeit. Nach über 40 Jahren erließ die UNESCO eine neue weltweite Empfehlung: Die >Recommendation concerning the Protection and Promotion of Museums and Collections, their Diversity and their Role in Society<. Eine UNESCO Recommendation stärkt die Position von Museen erheblich und bietet Schutz vor Gewalt, Schließung 
oder wirtschaftlichem Bankrott. Die Bundesregierung bat mich, als deutscher Experte an der dreitätigen Sitzung bei der UNESCO in Paris teilzunehmen, was mir eine Ehre war. Vor Ort setzte ich mich erfolgreich für die Berücksichtigung öffentlicher Sammlungen in der Neufassung der Schutzbestimmung ein. Diese sollten ebenfalls in den Geltungsbereich der Empfehlung fallen, da es meiner Meinung nach in dieser Hinsicht keine großen Unterschiede mehr zu klassischen Museen gibt. Mit entsprechenden Einschränkungen und Abgrenzungen zu privaten Sammlungen konnte die finale Fassung ausformuliert, mit zufriedenstellendem Ergebnis umgesetzt und veröffentlicht werden.

Darüberhinaus standen Gutachten zur Umsetzung neuer EU-Richtlinien, wie der sogenannten >Public Sector Information Guideline $<$, an. Diese Richtlinie hätte bestimmt, dass alle mit öffentlichen Mitteln erhobenen Daten mit uneingeschränktem Zugang öffentlich bereitgestellt werden müssten. Für Museen und größere Sammlungen hätte dies den Wegfall des Einkommenszweiges durch die Vergabe von Abbildungsgenehmigungen bedeutet.

Doch nicht nur der finanzielle Aspekt war wichtig, auch die Nachverfolgung der Urheberrechte wäre durch solch einen Beschluss nicht mehr gewährleistet gewesen. In einer kritischen Stellungnahme wies ich auf die damit einhergehenden, erheblichen Schwierigkeiten hin.

Im Hinblick auf diese Problematiken wurde die Richtlinie in einer entsprechend abgeänderten Form umgesetzt. Für Museen bedeutete das konkret die Verpflichtung zur Herausgabe von angefragtem Bildmaterial, die jedoch gegen eine sogenannte Verwaltungsgebühr erfolgen kann.

Was ich des Weiteren bewirken wollte, war eine zusätzliche thematische Schwerpunktsetzung bei dem Motto des internationalen Museumstages. Die bisher umgesetzten Themen stellten meiner Meinung nach ein $\mathrm{zu}$ positiv gefärbtes Weltbild dar, wie >Museums make Friends< oder >International Heritage <. Daher schlug ich bei den June Meetings 20I5 ein handfesteres Motto, nämlich >Museums and Hard History<, vor. Die Aufgabe der Museen ist neben der Erhaltung und positiven Beeinflussung der Gesellschaft vor allem auch die Dokumentation schwieriger Zeiten.

Ich bin sehr stolz, dass die General Assembly sich auf das Motto >Museums and contested Histories< einigen konnten und somit deutlich machte, dass wir uns auch auf dieser Ebene mit eventuell problematischen Inhalten beschäftigen.

Auch die Neufassung der bestehenden Museumsdefinition war einer der Schwerpunkte meiner ICOM-Arbeit. Beim ICOM-Weltkongress in Kyoto 2019 wurde ein viel diskutierter Vorschlag zu einer neuen Definition gemacht. Nach breiter Auffassung beinhaltete dieser jedoch viele Aspekte, die zu zentriert auf die westliche Welt waren und es daher nicht sinnvoll wäre, diese in einer all- 
gemeinen Museumsdefinition festzuschreiben. The well-being of the planet und andere philosophische Ansätze mögen in ihren Grundgedanken Werten entsprechen, die ein Museum vermitteln soll. Allerdings müssen wir beachten, dass Museen weltweit mit einer neuen Museumsdefintion arbeiten, sich darüber definieren und gewährleistet sein muss, dass sie sich damit vor allem vor der Politik als unterstützenswerte Institution rechtfertigen können. Innerhalb eines Komitees von weltweit zwölf Mitgliedern befassen wir uns daher mit der Entwicklung einer universal funktionierenden Neufassung. Ein interessanter Aspekt für eine Erweiterung der Museumsdefinition wäre die Aufgabe von Museen, das gegenseitige Verständnis in der Gesellschaft, zwischen Staaten und Individuen $\mathrm{zu}$ vertiefen. In der bisherigen Museumsdefinition wird das so nicht thematisiert. Bei der Weiterentwicklung hoffe ich auch auf das Mitgliederforum, damit wir bei der nächsten Generalkonferenz in Prag 2022 eine gute, fortgeschriebene Definition präsentieren können.

Auf nationaler Ebene schlug zu dieser Zeit der sogenannte Schwabinger Kunstfund hohe Wellen. Die Wiederentdeckung von angenommen in der NSZeit geraubten Kunstwerken fachte die Debatte über den Umgang mit und die Rückgabe von verfolgungsbedingt entzogenem NS-Raubgut wieder an. Als Gründungsmitglied des Komitees Kulturerbe innerhalb des deutschen Kulturbundes, initiierte ich diese Diskussion ohne Verzögerung auch bei ICOM Deutschland.

Außenpolitisch betrachtet baute sich massiver Druck auch durch den Jewish World Congress und die Commission for Material Claims against Germany auf. Die Sachlage wurde jedoch teils überspitzt dargestellt, denn Deutschland bemühte sich seit langem und dauerhaft sehr wohl um die Aufarbeitung dieses Aspekts seiner Geschichte, doch die Bearbeitung von Restitutionsansprüchen ist kompliziert. Viele Kunstwerke wurden nach dem Ende des Zweiten Weltkrieges am Arts Collecting Point im heutigen Zentralinstitut für Kunstgeschichte in München gesammelt und an ihre Besitzer oder deren Erben zurückgegeben. Dennoch blieb eine umfangreiche Liste an unauffindbaren Objekten und Objekten mit unklaren Besitzansprüchen bestehen. In der Washingtoner Erklärung verständigte man sich schließlich darauf, die Suche in Deutschlands öffentlichen Sammlungen fortzusetzen.

Im Zuge der neu entflammten Debatte berief BKM Frau Grütters in Berlin die »Taskforce Schwabinger Kunstfund«, die sich mit dessen Sichtung und eventuellen Restitutionsansprüchen, einem sehr zeitaufwändigen und komplizierten Unterfangen, beschäftigte. Mit zunehmender Dauer wuchs der externe Druck auf die Mitarbeiterinnen und Mitarbeiter der Taskforce trotz guter Arbeit, da sich eine breite internationale Öffentlichkeit auch in dieser Angelegenheit eine schnellere Vorgehensweise erhoffte. 
Aus persönlicher Überzeugung versuchten mein Vorstandskollege Matthias Henkel und ich, innerhalb von ICOM ein internationales Fachkomitee für Provenienzforschung $\mathrm{zu}$ gründen, womit wir jedoch scheiterten. Was wir in diesem Bezug auf den Weg bringen konnten, war eine Handreichung zur Provenienzforschung, die kleineren und mittleren Museen Hilfestellung bieten sollte. Im Endstadium der Planungen für die Veröffentlichungen der Handreichung intervenierte B KM, da der Leitfaden ihrer Meinung nach beim Deutschen Zentrum für Kulturgutverluste erscheinen sollte. Dies hatte zur Folge, dass die Fertigstellung des Leitfadens für Provenienzforschung aufgrund vieler neuer Partner drei Jahre länger dauerte und schließlich erst im Jahr 20I9 erschien. Mittlerweile wurde eine englische Übersetzung veröffentlicht und damit nicht zuletzt ein internationales Zeichen gesetzt, dass Deutschland diese Thematik durchaus ernst nimmt.

Auch in Nordrhein-Westfalen wurden Kunstwerke »wiederentdeckt «. Im Zuge des Insolvenzverfahrens der dortigen Landesbank wurden im Spielkasino Aachen frühe Werke von Andy Warhol gefunden, die im Ausland versteigert werden sollten. Um dies zu verhindern, bildete sich erneut ein Adhoc-Komitee im deutschen Kulturrat zur Frage des Verkaufes von Kulturgut aus öffentlichem Besitz. Neben der Veröffentlichung von Stellungnahmen nahmen wir dies vor allem zum Anlass, unsere Expertise in die von der Europäischen Union vorgegebene Novellierung des Kulturgutschutzgesetzes einzubringen. Jedes europäische Land war dazu angehalten, eine Neufassung des betreffenden Gesetzes unter Einhaltung bestimmter Eckpunkte vorzulegen, die Kunstwerke durch gewisse Wert- und Altersgrenzen entsprechend schützt und generell den Umgang mit Kulturgut umfassend regelt.

Dieses Verfahren beinhaltete unglaubliche viele Fallstricke. Die Organisation des internationalen Leihverkehrs oder Herkunftsfragen von Objekten waren wichtige Aspekte, die nicht verkompliziert oder gar außer Acht gelassen werden durften. $\mathrm{Zu}$ meiner Freude wurden neben Verwaltungsjuristen auch wir als Museumsexperten von ICOM zu Gutachten, schriftlichen Stellungnahmen und öffentlichen Anhörungen aufgefordert und konnten so unsere fachlichen Belange vertreten. Ein aufwendiger Prozess, an dem wir uns positiv und intensiv beteiligten, sodass dieser am Ende ein durchaus achtbares Ergebnis zeigte.

Schon mein ganzes Berufsleben lang und somit auch bei ICOM ist die Sicherung und Wahrung des kulturellen Erbes ein bestimmendes Thema meiner Tätigkeiten. Mir ist es ein großes Anliegen, wie innerhalb der gesellschaftlichen Entwicklung Kulturerbe als konstituierendes Grundelement einer jeden Gesellschaft in allen möglichen Ausprägungen und Ausformungen in Wert gehalten werden und in Wert gesetzt werden kann. 
Uns steht großes materielles und immaterielles Erbe zur Verfügung und wir müssen dafür sorgen, dass es in einem größeren, weltweiten kulturgeschichtlichen Zusammenhang vermittelt und verstanden wird. Fachtagungen wie etwa zu neuen Restaurierungs- und Konservierungstechniken, sowie zum Umgang mit Kulturgut und zur Ethik des Sammelns sollten dieses Bewusstsein unserer Museumskolleginnen und -Kollegen schärfen.

Auf den Tagungen beschäftigten wir uns mit allgemeinen ethischen Fragen zu Museumssammlungen, aber auch praktische Themen wir Richtlinien für Depotbau und -bestückung wurden erörtert.

Auch die Inklusion im weitesten Sinne war zu meiner Amtszeit Thema. Nicht nur für eindeutig im Sehen, Gehen und Verstehen behinderte Menschen, auch die Inklusion von kulturfernen Gruppen beschäftige unseren Vorstand.

Ebenso der Umgang mit Asylbewerbern, Flüchtlingen, Vertriebenen, denen wir gerne unsere Werte und unser kulturelles Erbe vorstellen und es in Bezug zu dem ihren setzen wollten. Wir entwarfen weg vom eurozentrischen Denken erfolgreiche und weniger erfolgreiche Strategien und mussten letzten Endes feststellen, dass sich diese aufgrund der verschiedenen Ausführungsmöglichkeiten und örtlichen Gegebenheiten stark voneinander unterscheiden können. Der wichtigste, universal geltende Aspekt ist jedoch stets das Vertiefen des gegenseitigen Verständnisses.

All diese Dinge nahm ich aktiv wahr, andere stieß ich wiederum an. Etwa die Jahreskonferenz in Schweden im vorletzten Jahr. Eine Kooperation mit ICOM Dänemark, Schweden, Norwegen, Island, Finnland und den Faröer Inseln, deren intensive Vorbereitungen noch in meiner Amtszeit begannen. Der Vorstand und die amtierende Präsidentin, Frau Prof. Reifenscheid, führten diese zu meiner Freude erfolgreich weiter und organisierten eine wunderbare Tagung in Schweden.

\section{Wie denken Sie sollte man bei ICOM Deutschland heute mit den Herausfor- derungen umgehen?}

Im Moment ist die Digitalisierung ein großes Generalthema. Museen in der digitalen Welt, neue Angebote, neue Positionen, die, extrem gesprochen, die Frage in den Raum stellen, ob das Original aufgrund von umfassender Verfügbarkeit von Reproduktionen seinen Wert verliert. Ich bin jedoch der Meinung, dass die Aura des Originals und das Original selbst noch lange wirksam sein werden. Somit müssen wir das Original und die neuen Möglichkeiten der Digitalisierung in einer positiven Art und Weise, auch zusammen mit den unterschiedlichen Nutzbarkeiten des Kulturgutschutzgesetzes, in einem sinnvollen und nicht zu komplizierten Gesamtkonzept miteinander verbinden. Vor allen 
Dingen muss für jede einzelne Institution neben allen Richtlinien immer ausreichender Freiraum der Gestaltung offen bleiben.

Allgemein sollten wir uns immer die Frage stellen, wie wir Neuerungen positiv beeinflussen und unser vorhandenes Kulturerbe schützen, in Wert setzen und erhalten können. Die Diskussion darüber mit der Politik muss weiter aktiv geführt werden, um immer wieder um Verständnis und Unterstützung für die Museen und Sammlungen zu werben, da Museen als Zuschussbetriebe »nur« über die Umwegrendite Geld in die öffentlichen Kassen bringen.

Um dies zu erreichen, sollte ICOM Deutschland vor allem national sichtbarer werden, weiterhin Handreichungen veröffentlichen und Standards setzen.

\section{Wie sahen Sie die Zusammenarbeit mit der Zentrale in Paris damals und aus heutiger Sicht?}

Mit Hans-Martin Hinz als Weltpräsident gestaltete sich die Zusammenarbeit über lange Zeit hinweg sehr gut. Auch die Referate dort waren mit Personen besetzt, die, nicht zuletzt aufgrund unserer Mitgliederstärke, stets den Kontakt zu uns suchten, sodass es nie Schwierigkeiten gab.

Die amtierende ICOM-Weltpräsidentin - die türkische Kollegin Suay Aksoy $^{30}$ - ist eine sehr fähige Frau, die das deutsche Nationalkomitee sehr schätzt.

So gesehen arbeiteten wir bestens mit der Zentrale in Paris zusammen, mit der Geschäftsstelle in Berlin sowieso.

\section{Welche Versäumnisse sehen Sie aus heutiger Sicht?}

Zwar reagierten wir innerhalb des Verbandes mit der Handreichung zur Provenienzforschung schnell und effizient, leider wurde deren Veröffentlichung durch die Zusammenarbeit mit dem Deutschen Zentrum für Kulturgutverluste verlangsamt.

Ansonsten konnten wir durch unsere internationale Vernetzung im Verband stets frühzeitig auf gesellschaftliche Konflikte reagieren.

Kulturpolitische Handlungen stehen natürlich immer in gesellschaftlichem Kontext, weshalb wir uns auch bei ICOM Deutschland bemühen, diesen aufzugreifen. Was in der Zukunft passiert, wissen wir leider nicht, ansonsten würden wir uns natürlich auch darum bemühen, entsprechende Vorkehrungen zu ergreifen.

Bei aktuellen Themen wie etwa der Digitalisierung oder der Inklusion leisten auch die jetzigen Vorstandsmitglieder gute Arbeit. Gleichzeitig verfolgen sie aufmerksam die Entwicklungen der modernen und zeitgenössischen 
Kunst, um einschätzen zu können, in welche Richtung sie geht. Dies wird vor allem bei der Bewertung von Sammlung, Kunst und Kunstwerken, sowie Künstlern wichtig. Auch wir haben eine Mitverantwortung für die wahnsinnig diffizile Entwicklung des Kunstmarktes, die wir jedoch mit höchster Vorsicht und Sensibilität wahrnehmen müssen.

\section{Konnten Sie in Ihrer Amtszeit etwas nicht umsetzen?}

Was leider nicht umgesetzt wurde, war die Idee, in Form einer Patenschaft Mitgliedsbeiträge von Kolleginnen und Kollegen aus den finanziell schwächer aufgestellten afrikanischen Nationalkomitees zu übernehmen.

Wegen Finanzierungsfragen lehnte der Vorstand diesen Vorschlag ab, weshalb darüber nie auf einer Mitgliederversammlung diskutiert wurde.

Das Spezialkomitee für Provenienzforschung scheiterte ebenfalls an den zu durchlaufenden Instanzen. Es bestanden bereits 36 Spezialkomitees, die alle aus einem Haushaltstitel finanziert wurden, weshalb ein neues Komitee von Anfang an kritisch gesehen wurde. Des Weiteren kristallisierte sich im Vorfeld der fehlende Bezug vieler Nationalkomitees zu dieser Thematik heraus, die vor allem Deutschland und bestenfalls noch Österreich und die Schweiz betraf. In anderen Ländern wären wiederum andere Spezialkomitees, beispielsweise gegen Gewaltherrschaften und Diktaturen nötig gewesen.

Auch die nationale Reichweite von ICOM Deutschland konnte ich nicht wie gewünscht verbessern. Trotz Newsletter und Pressemitteilungen erhielten wir über das Fachpublikum hinaus keine größere Resonanz. In Sachen öffentlicher Wahrnehmung gibt es also auch heute noch viel zu tun.

\subsection{Kommentierung durch die Interviewerin}

Das Interview mit Dr. Michael Henker fand in den Räumlichkeiten der Sudetendeutschen Stiftung in München statt. Die Terminfindung nahm einige Zeit in Anspruch, da Dr. Henker noch höchst engagiert im Aufbaustab selbiger Stiftung tätig war. Zudem kamen die Corona-Krise und etwaige Ausgangssperren dazwischen. Nach kleinen Startschwierigkeiten war Dr. Henker mehr als bereit, über seine Amtszeit zu sprechen. Den Fragenkatalog hatte er bereits vorliegen und so monologisierte er größtenteils ununterbrochen. Der Verlauf war erfolgversprechend. Nach dem Interviewtermin sollte jedoch sogleich die nächste Konferenz folgen, weshalb für Rückfragen wenig Zeit blieb. Größtenteils wurden die Fragen allerdings ohnehin im Gesprächsverlauf beantwortet.

Dr. Michael Henker beschrieb seine Amtszeit sowohl national als auch international als sehr turbulent. Dies wird durch die vielen verschiedenen 
»Baustellen«, an denen er gleichzeitig arbeitete, nachvollziehbar. Neben der Darstellung seiner Erfolge erzählt er auch ungehemmt von gescheiterten Vorhaben, wie es in dieser Form in bisherigen Interviews nicht der Fall war. Ob dies daran lag, dass es keine gescheiterten Vorhaben gab, ist eine Frage, die im Nachhinein leider offen bleibt. Auf die Frage nach dem jetzigen Umgang mit Herausforderungen im musealen Kontext, die der amtierenden Präsidentin begegnen, reagiert er mit Ratschlägen, wie Neuerungen gelingen, positiv aufgenommen und angenommen werden können.

In der Gesamtheit ist das Gespräch als offen und informativ zu bewerten. Die Interviewsituation war bis auf die Schlussminuten entspannt. Lediglich die Terminfindung und die Nachbereitung des Interviews waren etwas kompliziert. Durch sein Engagement in der Sudetendeutschen Stiftung, die Dr. Henker viel Zeit kostete, wurde die Sichtung des Interviews (weit) nach hinten verschoben. Die Überarbeitung seinerseits war allerdings als sehr positiv anzusehen, da es zu keinen nennenswerten Veränderungen des Narrativs oder Auslassungen beziehungsweise Streichungen kam. Einige ergänzende Zusätze vervollständigten das Interview. 
